\title{
Measles vaccine coverage estimates in an outbreak three years after the nation-wide campaign in China: implications for measles elimination, 2013
}

Chao $\mathrm{Ma}^{1+}$, Fangjun $\mathrm{Li}^{2+}$, Xiang Zheng ${ }^{3}$, Hong Zhang ${ }^{2}$, Mengjuan Duan ${ }^{1}$, Yanhua Yang ${ }^{2}$, Lixin Hao ${ }^{1}$, Qiru Su', Lance Rodewald ${ }^{4}$, Bosong Guo ${ }^{5}$, Shanliang Xiao ${ }^{6}$, Huaqing Wang ${ }^{1}, \mathrm{Li} \mathrm{Li}^{1}$, Junhua $\mathrm{Li}^{2}$, Huiming Luo ${ }^{1 *}$ and Lidong $\mathrm{GaO}^{2^{*}}$

\begin{abstract}
Background: China is approaching measles elimination, but indigenous measles still circulates. County L in China has reported measles-containing vaccine (MCV) coverage rates $>95 \%$ since 2000. Despite high reported coverage, a large measles outbreak occurred among young children in L County. We measured MCV coverage using 5 different methods during an investigation on this outbreak and compared our estimates with reported rates.

Methods: Reported coverage rates are determined by aggregating clinic-based data across the county: doses administered in each clinic divided by the number of children registered in each clinic. Our methods estimated coverage for the 2010-2012 birth cohort, and were (1) administrative method: doses administered in clinics divided by the birth cohort recorded in the Statistical Year Book, (2) house-to-house convenience-sample survey of children living near cases, (3) vaccination clinic records review, (4) determination of a convenience sample of measles outbreak cases' vaccination statuses and using the field vaccine efficacy outbreak equation to estimate population coverage, and (5) a seroprevalence survey using a convenience sample of residual blood samples from hospitals.

Results: The measles outbreak totaled 215 cases, representing an incidence of 195.8 per million population. Our estimated MCV coverage rates were: (1) administrative method: $84.1 \%-87.0 \%$ for MCV1 and $80.3 \%-90.0 \%$ for MCV2, (2) in-house survey: $83.3 \%$ of 9-17 month children received MCV1, and 74.5\% of 24-47 month children received MCV2, (3) clinic record review: $85.5 \%$ of 9-17 month children received MCV1, and $73.2 \%$ of $24-59$ month children received MCV2, (4) field VE method: $83.6 \%$ of 9-47 month children received one or more MCV doses, and (5) serology: seropositive rates were $<80 \%$ in the $12-17$ and $18-23$ month age cohorts.

Conclusions: Compared with reported coverage $>95 \%$, our 5 coverage assessments all showed substantially lower coverage. China should evaluate guidelines for reporting vaccination coverage and identify feasible improvements to the assessment methods.
\end{abstract}

Keywords: Measles, Vaccination, Coverage, Estimation, Elimination, China

\footnotetext{
* Correspondence: Luohm@Chinacdc.cn; gldlj@hotmail.com

${ }^{\dagger}$ Equal contributors

'National Immunization Programme, Chinese Center for Disease Control and

Prevention, 27 Nanwei Road, Xicheng District, Beijing, China

${ }^{2}$ Hunan Province Center for Disease Control and Prevention, Changsha, Hu

Nan province, China

Full list of author information is available at the end of the article
} 


\section{Background}

The World Health Organization Western Pacific Region has been striving to eliminate measles since 2005 [1] and set a goal to eliminate measles in the region by 2012 . China adopted this goal and endorsed an action plan for measles elimination in 2006 that included continuing a two-dose measles-containing vaccine (MCV) strategy (administered at 8 months and 18-23 months of age), and called for routine measles vaccine coverage to be greater than $95 \%$ for both doses in every county, while using supplementary immunization activities (SIAs) to close immunity gaps among children [2].

Between 2004 and 2009, 27 of the 31 mainland provinces conducted unsynchronized province-wide measles SIAs targeting children aged 8 months through 14 years. In September 2010, China conducted a synchronized nationwide SIA targeting different age groups by province; children were vaccinated regardless of their prior vaccination status. Using the combined province-wide catch-up SIAs and nationwide follow-up SIA strategy, each province had covered their 1995-2009 birth cohorts through SIAs by 2010.

With these efforts, the measles incidence of China decreased dramatically, from 99.4 per million population in 2008 to 4.6 in 2012. However, indigenous measles virus outbreaks have been continuously reported, and a resurgence occurred in the end of 2012 that continued into 2013. Between January and October 2013, there were over 26,000 measles cases reported in China, which was 1.7 times more than case number of the same time period in 2011 and 4.6 times more than that in 2012. Of these cases, $68 \%$ were among children under 5 years of age [3], raising the concern that timely, 2-dose MCV coverage may not be high enough to eliminate measles outbreaks among young children.

In China, all vaccines are delivered through a public infrastructure with vaccination clinics and fixed-sites in urban and rural areas, and are offered to local children and to children new to the area who have registered for clinic services. Clinic immunization providers record each dose of vaccine administered, either through a vaccination card or a computerized immunization information system. Children who receive any routine vaccination in the clinics are registered in the clinic. Clinics are required to report vaccination coverage levels by dividing the number of children vaccinated in the clinics by the number of children registered in clinics. Reported coverage measured with this method has consistently been $>98 \%$ in China [4,5], however, these rates may be too high because children not registered in a clinic are not included in the denominator. Survey-based coverage estimates in China have been lower than reported coverage. For example, a 2009 estimate showed coverage to be $91.1 \%$ for MCV1 and $84.3 \%$ for MCV2 [2]. A national coverage survey was conducted in
2011, using a stratified cluster sampling to select counties and Probability Proportional to Size sampling to select objects. Of the 4,681 children randomly sampled from 32 counties, 160 townships, and 480 villages, coverage rates for MCV1 and MCV2 were $99.4 \%$ and $93.35 \%$, respectively [6].

In February 2013, a measles outbreak was reported in L County of $\mathrm{Hu}$ Nan province, with 215 measles cases, representing an incidence of 195.8 per million population. This county has reported administrative coverage of MCV greater than 95\% since 2000. As a part of the provincewide catch up SIA in April 2009, L County targeted 8month through 14-year-old children (birth cohorts of 1994 through part of 2008), and reported 98\% coverage. As part of the 2010 nation-wide campaign, L County targeted 8-month through 4-year-old children (birth cohorts between October 2005 and 2009) and reported 98\% coverage. Among all cases reported in the 2013 outbreak, 88\% were among $\leq 3$ year old children, and only $22 \%$ of $8-47$ months old cases had received at least $1 \mathrm{MCV}$ dose. The disease pattern raised our concern that there may be a discrepancy between reported and real MCV coverage rates.

The investigation of this outbreak provided an opportunity to determine whether coverage was as high as had been reported. We used 5 qualitatively different methods to estimate MCV coverage or protection levels in $\mathrm{L}$ County. We report the results of these measurements and contrast them to the reported coverage rates.

\section{Methods}

Case-based measles surveillance data were obtained from the National Measles Surveillance System. A suspected measles case was defined using World Health Organization (WHO) criteria as a person with fever, rash, and one or more of the following symptoms: cough, coryza, or conjunctivitis. Key variables obtained included age, sex, address of residence, date of onset, occupation, MCV vaccination history, and measles-specific immunoglobulin (IgM) testing results. Case data were analyzed to describe person, place and time characteristics of the outbreak. During the field investigation, additional data were collected to estimate measles vaccine coverage and population susceptibility by using the following five methods.

\section{Administrative data coverage estimate}

Routine immunization MCV1 and MCV2 coverage of the birth cohorts born after the 2010 measles SIA were estimated by using data from L County. The number of MCV1 and MCV2 doses administered through routine immunization in L County clinics in 2010, 2011 and 2012 were used as numerators, and the published data on number of new births reported in the Statistical Year Book for L County as denominators. Because this method is ecologic, the number of MCV2 doses can exceed the number 
of MCV1 doses when children change clinics. This method differs from the reported coverage method in the denominator - the reported coverage uses children registered in clinics, while this administrative data coverage estimate uses recorded births.

\section{House-to-house coverage survey}

During the measles outbreak investigation, we identified a sample of children who had not acquired measles but who lived near a convenience sample of the measles cases. Investigators started from the house closest to the residence of each case, and visited additional houses one-by-one in a random direction to search for three healthy children of the same age as the case. Investigators reviewed vaccination certificate cards, and obtained records of each dose of MCV administered. If the certificate card was not available during in-house visit, investigators checked the immunization records in the vaccination clinic. In total, 190 parents of children aged 9 months to 15 years old were interviewed and their children's vaccinations recorded.

\section{Immunization clinic records review}

In the 9 townships of L County with the most measles cases reported, investigators reviewed clinic-registered children's vaccination records. Records from a minimum of 40 children, 9-59 months old (birthdate between 1 January 2008 and 31 March 2012), were randomly selected from each township clinics' list of registered children, and all MCV doses administered were recorded for each child.

\section{Coverage estimation by vaccine efficacy equation}

In the 1980s, Orenstein and colleagues developed an equation to determine vaccine efficacy (VE) in the field during an outbreak [7]. The equation is PCV $=\left(\mathrm{PPV}-\left(\mathrm{PPV}^{*} \mathrm{VE}\right)\right) /$ $(1-(P P V * V E))$, where PCV is the proportion of cases vaccinated; PPV is the proportion of the population vaccinated; and VE is vaccine efficacy. PPV (coverage) can be calculated if PCV is known and the vaccine has a known, relatively constant VE. We estimated MCV coverage of 947 months old children using the algebraic transformation of the field VE equation: PPV $=\mathrm{PCV} /(1-\mathrm{VE}(1-\mathrm{PCV}))$ $[7,8]$. In this calculation, we assumed efficacy of a singledose of measles vaccine to be $90 \%$, and we assumed that the vaccination rate in case-patients with unknown vaccination history was the same as in case patients with known vaccination history [8].

\section{Prevalence of measles antibodies study}

There are 2 major hospitals in L County, and in March 2013, we obtained residual serum specimens from these 2 hospitals' clinical laboratories. Specimens were from non-fever, non-rash ill patients less than 35 years old.
The specimens were shipped to $\mathrm{Hu}$ Nan Provincial CDC for measles-specific IgG testing to determine susceptibility to measles. The commercial Virion $\backslash$ Serion ELISA kit was employed to detect and quantify human IgG antibodies against measles virus in the sera. The laboratory results were interpreted according to the manufacturer's instructions. Titers $\geq 1: 200$ were considered positive and titers $<1: 150$ were considered negative. Sera samples for which the results were equivocal were retested using the same method; if again equivocal, we considered the result negative. Demographic information including age, gender, and location of residence were obtained, but individual-identifying data and immunization status and history of measles infection were not obtained. These data were analysed after determining sero-positivity and geometric mean titer.

\section{Ethical review considerations}

Individual identifying information on the persons whose blood was tested for measles antibodies was not obtained by the investigators; the individuals were not contacted or interviewed; and informed consent was therefore not obtained. Obtaining and testing these samples of residual blood was approved by the Ethical Review Committee of Chinese Center for Disease Control and Prevention (Approval Notice No. 201410), recognizing that the right and the welfare of the subject are adequately protected; the potential risks are outweighed by potential benefits. Administrative (doses administered) data, coverage survey data, and vaccination record review data that are collected as part of a vaccine preventable disease outbreak investigation are considered by China CDC's Ethical Review Committee to be exempt from IRB review. Informed consent is not obtained for accessing administrative, survey, and immunization clinic record data. Individual identifying data were not retained in analytic data sets.

\section{Results}

\section{Outbreak description}

A total of 215 measles cases were identified in 24 of 26 townships in L County; 209 were laboratory confirmed and 6 were epidemiologically linked to a laboratory confirmed case. Of these, 153 cases (71.2\%) were male, representing incidence (per million population) of 243.9 for male and 107.1 for female in this county. The outbreak started on January 9 and ended on June 19, 2013 (Figure 1). Of the 215 cases, 189 cases $(87.9 \%)$ were born after 1 January 2010 and therefore had not been part of the 2010 nationwide measles campaign; 16 cases (7.4\%) were born between 1 October 2005 and 31 December 2009 and were part of the 2010 nationwide campaign target age group; and 10 cases (4.6\%) were $>15$ years old (Figure 2). 


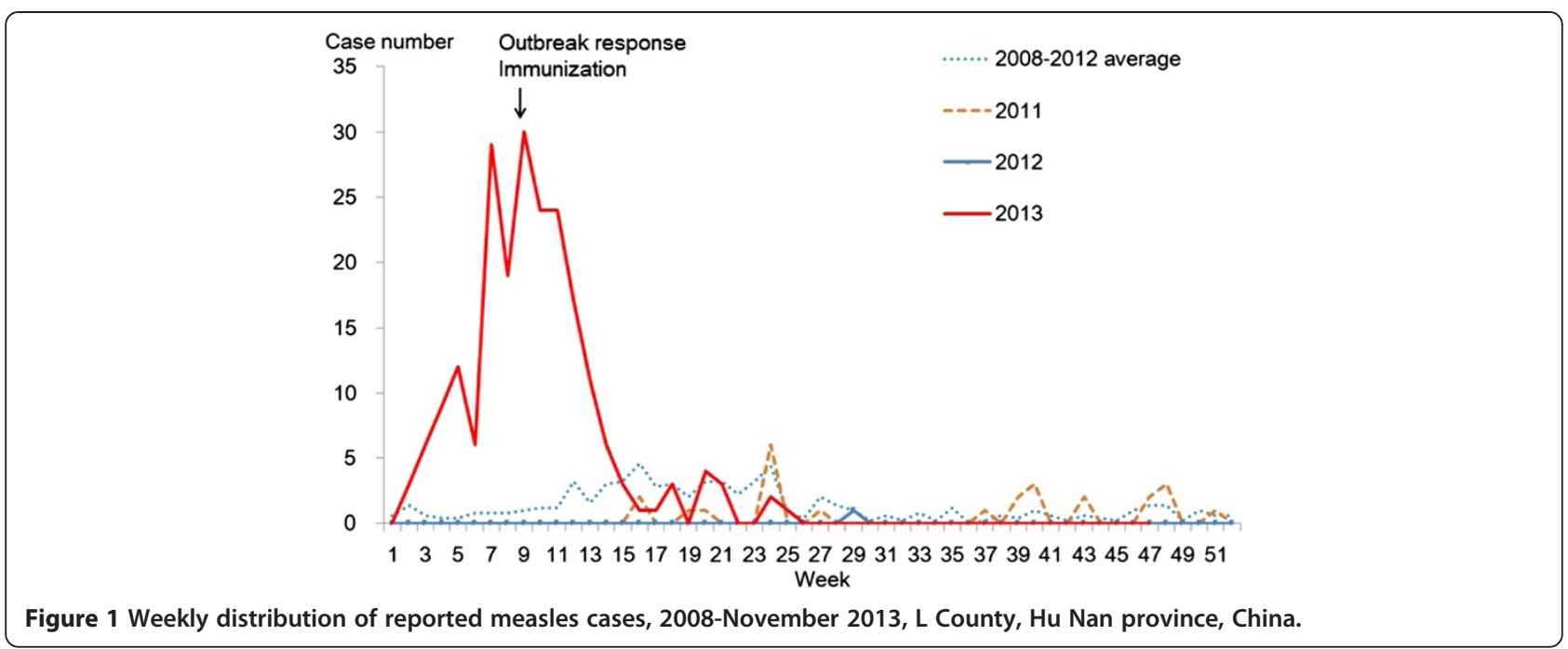

\section{Coverage estimation}

From 2010 through 2012, the annual number of doses of MCV1 delivered through routine immunization in $\mathrm{L}$ County ranged between 13,718 and 14,452 doses, and the annual number of MCV2 doses ranged between 13,874 and 14,711 doses. Officially reported administrative coverage data for both MCV1 and MCV2 were >99\% (Table 1).

The number new births in 2010 to 2012 reported in the Statistical Year Book ranged between 16,235 and 16,857 . The calculated coverage estimate using the above doses administered data as the numerator and reported births as the denominator in 2010 through 2012 in L County ranged from $84.1 \%$ to $87.0 \%$ for MCV1, and from $80.3 \%$ to $90.0 \%$ for MCV2 (Table 1).

For the house-to-house survey, investigators interviewed 186 parents of children aged 9-179 months. Of 72 children aged 9-17 months who were age-eligible for MCV1, 83.3\% (95\% CI: 72.7-91.1) had received the first dose. Of 28 children between 18 and 23 months of age and eligible for MCV2, 85.7\% (95\% CI: 67.3-96.0) received MCV1, and 32.1\% (95\% CI: 15.9-52.4) received MCV2. Among 51 children 24-47 months old and due

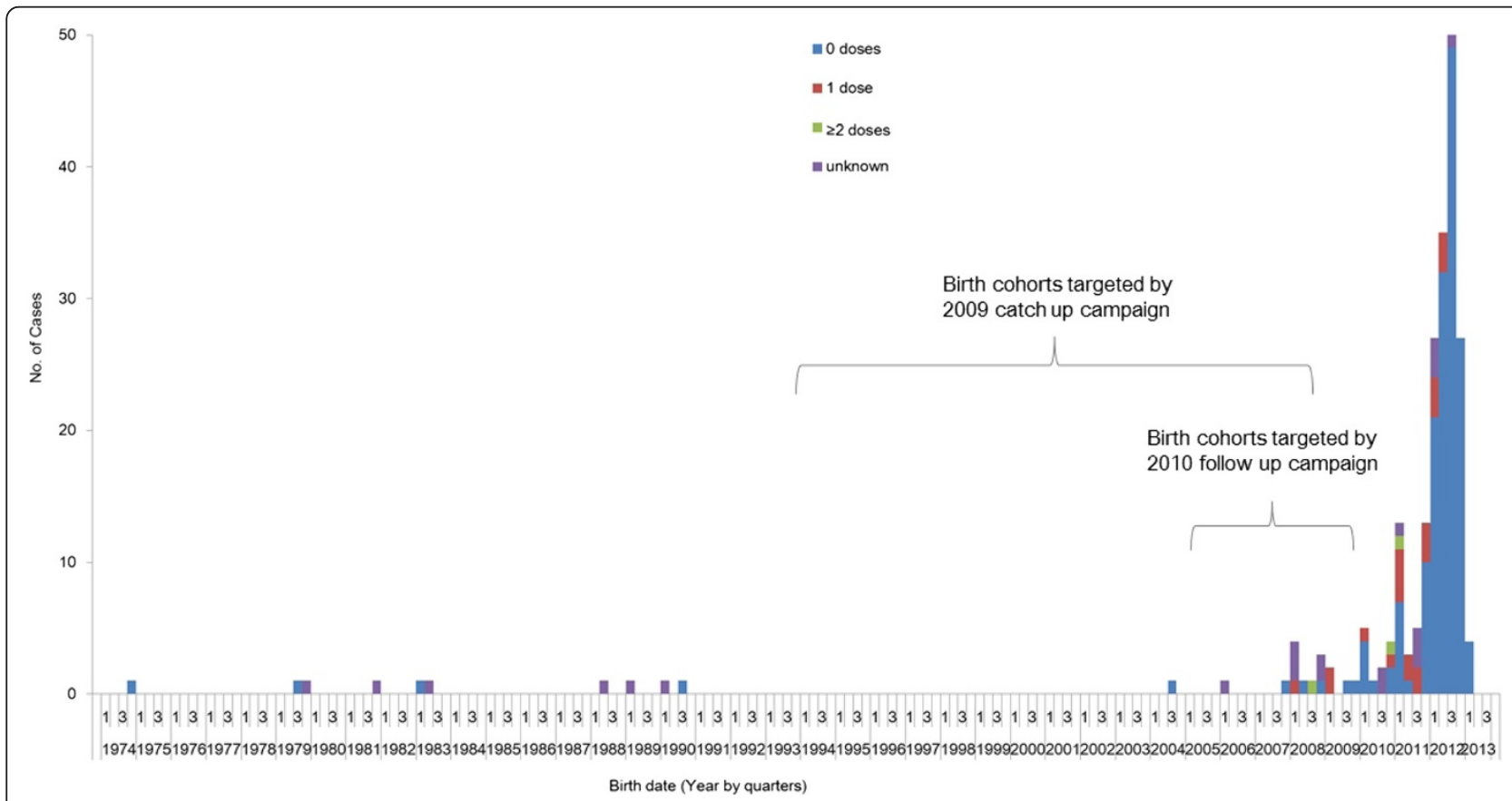

Figure 2 Birth-date distribution of the 215 measles cases reported in L County, Hu Nan province. 
Table 1 Estimated MCV routine immunization coverage during 2010-2012, L County, Hu Nan province, China

\begin{tabular}{|c|c|c|c|c|c|c|}
\hline \multirow[t]{2}{*}{ Year } & \multirow[t]{2}{*}{ Dose } & \multicolumn{3}{|l|}{ Reported coverage } & \multicolumn{2}{|c|}{ Administrative estimated coverage } \\
\hline & & Number of vaccinated $^{\ddagger}$ & Number of target ${ }^{\dagger}$ & Reported coverage (\%) & Number of new birth cohort & Estimated coverage (\%) \\
\hline \multirow[t]{2}{*}{2010} & MCV1 & 14452 & 14526 & 99.5 & 16613 & 87.0 \\
\hline & MCV2 & 14657 & 14740 & 99.4 & 16725 & 87.6 \\
\hline \multirow[t]{2}{*}{2011} & MCV1 & 13718 & 13728 & 99.9 & 16235 & 84.5 \\
\hline & MCV2 & 14711 & 14722 & 99.9 & 16354 & 90.0 \\
\hline \multirow[t]{2}{*}{2012} & MCV1 & 14182 & 14193 & 99.9 & 16857 & 84.1 \\
\hline & MCV2 & 13874 & 13886 & 99.9 & 17267 & 80.3 \\
\hline
\end{tabular}

FThe number of MCV2 doses can exceed the number of MCV1 doses when children change clinics.

†The "number of target" here, is the total number of children who have registered, and received routine immunization in vaccination clinics. It's the denominator of administrative coverage.

for MCV2, 98.0\% (95\% CI: 89.5-99.9) received MCV1, and 74.5\% (95\% CI: 60.4-85.7) received MCV2. All 35 children over 48 months old had received MCV2 (Table 2).

In L County immunization clinics, 365 children's immunization records were identified at random and were reviewed. Among 69 children aged 9-17 months, 85.5\% (95\% CI: 75.0-92.8) received MCV1. Among 46 children aged 18-23 months (eligible for MCV2), all received MCV1, and 41.3\% (95\% CI: 27.0-56.8) received MCV2. Among the 250 children aged 24-59 months, 73.2\% (95\% CI: 67.3-78.6) received MCV2 (Table 3).

For the VE equation method, we obtained MCV vaccination status for 108 measles cases aged 9-47 months who were reported in the outbreak. Among these, 22 (20.4\%) had received $\geq 1$ dose MCV, while 86 (79.6\%) had received no MCV doses. Using these rates to determine the proportion of cases vaccinated, and assuming $90 \%$ VE for one dose of measles vaccine, the vaccination coverage for this age group was estimated with the field $\mathrm{VE}$ equation to be $83.6 \%$.

\section{Prevalence of measles antibodies}

In total, 632 residual serum specimens were tested for measles IgG antibodies, and 74.5\% (95\% CI: 70.9-77.9) of these samples tested positive. Seropositive rates decreased from $60.0 \%$ (95\% CI: 45.2-73.6) in 0-1 month group to $18.2 \%$ (95\% CI: $5.2-40.3)$ in the $6-7$ month age group. Seropositivity increased starting with the 8-9 months group and reached $90.1 \%$ (95\% CI: 82.1-95.4) in the 24-47 month age group. Children 48 months to 15 years old had the highest seropositive rate, which was 95.8\% (95\% CI: 90.5-98.6). The 12-17 month and 18-23 month groups had seropositive rates less than $80 \%$. GMT titers for each age group showed similar trends as the seropositive rate (Table 4).

\section{Discussion}

We have shown that reported MCV coverage, which is determined using clinic-registered children as the denominator, yields a higher coverage estimate than 5 qualitatively different coverage assessment methods that were obtained during a measles outbreak investigation. Because officially reported coverage estimates in China do use clinic-registered children as the denominator, reported coverage is likely to be higher than actual coverage. This higher-than-actual reported coverage may provide a partial explanation why indigenous circulation of measles has continued in China despite more than 25 years of a 2-dose MCV vaccination policy, the strategy of using measles SIAs, and high reported coverage with MCVs.

Conducting SIAs is useful to address coverage inequities and rapidly close population immunity gaps in targeted age groups. This has been demonstrated both in China and elsewhere in the world [9-12]. However, SIAs should not be considered superior to routine immunization [13], because they provide vaccination in an intermittent manner, allowing for accumulation of susceptible children between campaigns. Experience from previous elimination programs has demonstrated that a 2-dose measles vaccine

Table 2 Coverage assessment from in-house interview, L County, Hu Nan province, China, 2013

\begin{tabular}{|c|c|c|c|c|c|c|}
\hline \multirow{2}{*}{$\begin{array}{l}\text { Age group } \\
\text { (months) }\end{array}$} & \multicolumn{3}{|c|}{ No. of children by MCV doses } & \multirow[b]{2}{*}{ Total } & \multirow{2}{*}{$\begin{array}{l}\text { Proportion of } \geq 1 \\
\text { doses }(\%, 95 \% \mathrm{Cl})\end{array}$} & \multirow{2}{*}{$\begin{array}{l}\text { Proportion of } \geq 2 \\
\text { doses }(\%, 95 \% \mathrm{Cl})\end{array}$} \\
\hline & 0 doses & 1 dose & $\geq 2$ doses & & & \\
\hline $9-17$ & 12 & 59 & 1 & 72 & $83.3(72.7-91.1)$ & - \\
\hline $18-23$ & 4 & 15 & 9 & 28 & $85.7(67.3-96.0)$ & $32.1(15.9-52.4)$ \\
\hline $24-47$ & 1 & 12 & 38 & 51 & $98.0(89.5-99.9)$ & $74.5(60.4-85.7)$ \\
\hline 48-179 & 0 & 0 & 35 & 35 & 100 & 100 \\
\hline Total & 17 & 86 & 83 & 186 & 90.9 (85.8-94.6) & - \\
\hline
\end{tabular}


Table 3 MCV immunization status for the 365 registered children, L County, Hu Nan province, China, 2013

\begin{tabular}{|c|c|c|c|c|c|c|}
\hline \multirow{2}{*}{$\begin{array}{l}\text { Age group } \\
\text { (months) }\end{array}$} & \multicolumn{4}{|c|}{ Number of children (No., \%) } & \multicolumn{2}{|c|}{ Coverage $(\%, 95 \% \mathrm{Cl})$} \\
\hline & 0 doses & 1 dose & 2 doses & Total & $\geq 1$ dose & $\geq 2$ doses \\
\hline $9-17$ & 10 & 54 & 5 & 69 & $85.5(75.0-92.8)$ & - \\
\hline $18-23$ & 0 & 27 & 19 & 46 & $100.0(92.3-)$ & $41.3(27.0-56.8)$ \\
\hline $24-59$ & 2 & 65 & 183 & 250 & $99.2(97.1-99.9)$ & $73.2(67.3-78.6)$ \\
\hline Total & 12 & 146 & 207 & 365 & 96.7 (94.3-98.3) & $56.7(51.5-61.9)$ \\
\hline
\end{tabular}

policy has been highly successful in achieving and maintaining measles elimination status [14-16]. In the United States, there have been three efforts to eliminate measles targeting 1967, 1982, and 1996. Over the years, the U.S. experienced several failures, but systematically incorporated the lessons learned from each failure into subsequent efforts, and finally achieved the goal in 2000 [17,18]. Key lessons learned from the efforts include: (1) elimination requires very high $\mathrm{MCV}$ vaccination coverage by age 2 years, (2) a second dose of measles vaccine is needed to achieve satisfactory levels of immunity, and (3) coverage assessment is crucial $[17,19]$.

In this outbreak, the 2010-2012 birth cohorts, which became age-eligible for measles vaccination following the 2010 campaign, had the highest incidence of measles. Fully $80 \%$ of the $108 \mathrm{MCV}$-eligible cases were unvaccinated. This pattern raised our interest in providing alternative estimates of true coverage as a check on the high reported coverage that was calculated using doses administered data with clinic-registered children as the denominator. Each result of the five independent measurement methods revealed that coverage of 2010, 2011, and 2012 birth cohorts was below 85\%, which is much lower than the $95 \%$ target objective needed to eliminate measles, and much lower than coverage that was officially reported.

Table 4 Sero-prevalence of measles by age group, L County, Hu Nan province, China, 2013

\begin{tabular}{lllll}
\hline $\begin{array}{l}\text { Age group } \\
\text { (months) }\end{array}$ & $\begin{array}{l}\text { No. of } \\
\text { tested }\end{array}$ & $\begin{array}{l}\text { No. of } \\
\text { positive }\end{array}$ & $\begin{array}{l}\text { Positive rate } \\
(\%, 95 \% ~ C I)\end{array}$ & GMT (IU/mL) \\
\hline $0-1$ & 50 & 30 & $60.0(45.2-73.6)$ & 163.05 \\
$2-3$ & 37 & 15 & $40.5(24.8-57.9)$ & 163.23 \\
$4-5$ & 34 & 10 & $29.4(15.1-47.5)$ & 43.27 \\
$6-7$ & 22 & 4 & $18.2(5.2-40.3)$ & 27.07 \\
$8-9$ & 20 & 5 & $25.0(8.7-49.1)$ & 34.25 \\
$10-11$ & 27 & 10 & $37.0(19.4-57.6)$ & 38.9 \\
$12-23$ & 71 & 54 & $76.1(64.5-85.4)$ & 360.16 \\
$24-47$ & 91 & 82 & $90.1(82.1-95.4)$ & 822.43 \\
$48-179$ & 120 & 115 & $95.8(90.5-98.6)$ & 914.99 \\
$180-419$ & 160 & 146 & $91.3(85.8-95.1)$ & 743.84 \\
Total & 632 & 471 & $74.5(70.9-77.9)$ & 359.45 \\
\hline
\end{tabular}

Several recently-published and unpublished studies also showed that low coverage of new birth cohorts after the 2010 campaign was a key factor contributing to China's measles resurgence [8]. These outbreaks were due to failure to provide measles vaccine for children at recommended ages. Although the 2011 national survey showed high coverage for both MCV1 and MCV2 at the national level, low-coverage areas were also identified in the survey [5], indicating that "pocket areas" exist. A recently-published coverage survey also showed that coverage among a migrating population was lower than among local populations [20].

To eliminate measles in China, learning from other elimination programs and from domestic measles outbreaks is critically important. Several strategies are important for China. First, increasing and maintaining high ( $\geq 95 \%$ ) coverage of both MCV1 and MCV2 through routine immunization is the top priority at this stage. The 1996-2009 birth cohorts (in some provinces back to the 1990 birth cohorts) have been targeted by SIAs, and many fewer cases have been reported from these age-group since 2011 [21]. The majority of new, potential susceptibles will come from new born children. Maintaining high coverage through routine immunization can minimize the number of susceptibles, and consequently avoid periodic epidemics. China is undergoing a large urban migration, leading to over 236 million migrant workers in 2012 [22], which complicates the administration of vaccines given in series and given during times of family movement. Conducting outreach to migrant children becomes an additional need for closing immunity gaps.

Second, delivering both MCV1 and MCV2 to 8month and 18-month old children on time is essential. Several studies indicated that children born to vaccinated mothers lose their maternal antibodies earlier than children whose mothers were immune through infection [23-25]. Seroprevalence data from this study also showed the trend of rapidly decreasing seroprevalence - from $60 \%$ in $0-1$ months to the lowest level of $18.2 \%$ in infants $6-7$ months of age. China provides MCV1 at one of the youngest ages globally -8 months. This young age is designed to protect children as early as possible, prior to risk of getting infection. To avoid future outbreaks, it is crucial to attain high coverage levels by timely vaccination [20], so that herd immunity 
can be robust enough to protect children too young to vaccinate.

Third, frequent monitoring of coverage is important to identify program areas that need to be strengthened. Outbreaks of measles can also be used to find program weaknesses, but outbreaks are lagging indicators. In contrast, coverage can be a leading indicator to find areas in need of additional effort before an outbreak occurs [17]. The unrealistically high coverage reported is not consistent with China's current measles epidemiology. Having higherthan-actual reported coverage can undermine confidence in vaccination strategies by giving false sense that the programme cannot improve coverage, and may lead to a lack of understanding why outbreaks are occurring.

Fourth, coverage assessments should strive to include all children in an area. A purpose of coverage measurement is to identify areas at risk of disease outbreaks. It would not have been possible to use reported coverage to predict the L County outbreak because reported coverage ranged from $99.4 \%$ to $99.9 \%$ for each of the birth cohorts affected by the outbreak. Most likely, the restriction of the denominator to clinic-registered children resulted in missing children in the area who should have been registered in the clinic and vaccinated. Given the large urban migration in China, including all children in an area in coverage assessments will be an important component in an effective measles elimination strategy. When a measles outbreak occurs in a community, an initial step for public health officials can be to use population estimates to determine whether the number of children registered in community clinics is consistent with population estimates. If inconsistent, community based surveys using rigorous methodology may be indicated [26].

Fifth, enhancing outbreak analysis and response activities to close immunity gaps will be needed to eliminate measles. Given the imbalance of socioeconomic development and immunization program capacity, small area SIAs are likely to be needed as a supplement to routine immunization in less developed areas. These SIAs depend on the epidemiology of measles and identification of immunity gaps. Surveillance and outbreak analysis can provide additional information to determine the extent to which missed opportunities to vaccinate occur, and then decide which types of actions should be taken [27].

\section{Limitations}

This study has several limitations, primarily the limitations of each of the 5 methods. The administrative method used Statistical Year Book birth records, and therefore would miss in- and out-migration among young children. The house-to-house survey is a convenience sample of neighborhood- and age-matched children, and is not representative of the entire county.
Since these children did not get measles despite living near cases, their coverage may have been higher than the county average. The clinic review will miss children who never registered with the clinic, and can include children who moved away from the area. The VE equation method relies on the stability of the VE estimate. The serological survey was a convenience sample of children seen in the 2 hospitals, and previous studies comparing commercially available EIA assay versus the gold standard plaque reduction-neutralization assay have demonstrated that EIA was less sensitive, but is a reliable identifier of measles-seronegative individuals [28]. Although each of the 5 methods used in this study is imperfect and has advantages and disadvantages, all the 5 results come to the same conclusion that actual coverage is lower than reported coverage that is determined with a clinic-based denominator. We feel that a key strength of this study is the convergence of results from 5 qualitatively different methods. We therefore believe that our conclusion is supported by the evidence provided.

\section{Recommendation}

We recommend review and evaluation of the methods for estimating officially-reported vaccination coverage levels in China, with a goal of identifying feasible coverage assessment methods that will provide useful information for the immunization program in China. Methods that include children not registered in immunization clinics should be sought.

\section{Conclusions}

The L County measles outbreak was due to low routine immunization coverage, which was inconsistent with the high coverage rates that had been reported before the outbreak. Despite very high reported administrative coverage, measles epidemics will occur as a result of actual low vaccine coverage in socio-demographically clustered, mainly unvaccinated communities that accumulate susceptibles in new birth cohorts. Improving coverage assessments to identify areas of low coverage in time to prevent outbreaks will help the program achieve the goal of eliminating measles.

\section{Abbreviations}

CDC: Center for Disease Control and Prevention; MCV: Measles containing vaccine; SIA: Supplementary immunization activities; VE: Vaccine efficacy; WHO: World Health Organization.

\section{Competing interests}

The authors declare that they have no competing interests.

Funding to support the field investigation activities described in this study came from national, provincial and local governments of China. The authors wrote this paper as part of their routine responsibilities as medical officers in the China Centers for Disease Control and prevention (CDC), and province level, prefecture level and county level CDCs. 


\section{Authors' contributions}

$C M, F L, H L, L G$ and $L R$ lead the study and the writing of this manuscript. $X Z$ LH and BG participated the in-house coverage survey. MD, YY, SX and QS conducted administrative data analysis and registered children survey. HW, $\mathrm{LL}$, JL lead the sero-prevalence survey. $\mathrm{HZ}$ conducted laboratory testing. $\mathrm{LR}$ also helped to draft the manuscript. All authors read and approved the final manuscript.

\section{Acknowledgements}

We gratefully acknowledge for the efforts made by staff of Hunan Province Center for Disease Control and Prevention (CDC), Longhui County CDC, and Shao Yang prefecture CDC who participated the field investigation and laboratory testing.

\section{Author details}

${ }^{1}$ National Immunization Programme, Chinese Center for Disease Control and Prevention, 27 Nanwei Road, Xicheng District, Beijing, China. ${ }^{2}$ Hunan Province Center for Disease Control and Prevention, Changsha, Hu Nan province, China. ${ }^{3}$ Taizhou Prefecture Center for Disease Control and Prevention, Taizhou, Zhe Jiang province, China. ${ }^{4}$ World Health Organization Office in China, Beijing, China. ${ }^{5}$ Longhui County Center for Disease Control and Prevention, Shaoyang, Hu Nan province, China. ${ }^{6}$ Shaoyang Prefecture Center for Disease Control and Prevention, Shaoyang, Hu Nan province, China.

Received: 27 October 2014 Accepted: 12 January 2015

Published online: 22 January 2015

\section{References}

1. WHO. Progress towards the 2012 measles elimination goal in WHO's Western Pacific Region, 1990-2008. Wkly Epidemiol Rec. 2009;84(27):271-9.

2. $\mathrm{Ma}$ C, An Z, Hao L, Cairns KL, Zhang Y, Ma J, et al. Progress toward measles elimination in the People's Republic of China, 2000-2009. J Infect Dis. 2011;204 Suppl 1:S447-54.

3. Ma C, Hao L, Zhang Y, Su Q, Rodewald L, An Z, et al. Monitoring progress towards the elimination of measles in China: epidemiological observations, implications and next steps. Bull World Health Organ. 2014;92(5):340-7.

4. WHO. Immunization: measles (MCV) by country. [http://apps.who.int/gho/ data/node.main.A826] (Accessed on 23 Jan 2015).

5. Wendi W, Dawei L, Lei C, Biao G, Lingsheng C, Huaqing W, et al. Study on the estimation of routine immunization coverage of China, 2005-2007. Chinese Journal of Vaccines and Immunization. 2009;15(4):363-6.

6. Lei $C$, Huaqing $W$, Jingshan Z, Ping Y, Lingsheng $C$, Guomin Z, et al. Coverage survey after expanded immunization program. Chinese Journal of Vaccines and Immunization. 2012;18(5):419-24

7. Orenstein WA, Bernier RH, Dondero TJ, Hinman AR, Marks JS, Bart KJ, et al. Field evaluation of vaccine efficacy. Bull World Health Organ. 1985;63(6):1055-68.

8. Gao J, Chen E, Wang Z, Shen J, He H, Ma H, et al. Epidemic of measles following the nationwide mass immunization campaign. BMC Infect Dis. 2013;13:139.

9. Liwei $Z$, Jianguo R, Yiren F, Haijun L, Shuxia L, Wei L, et al. Evaluation measles mass campaign of NingXia Hui Autonomous Region in 2005. Med Ani Pre. 2007;23(11):820-2.

10. Shuyan Z, Xiaofeng L, Dapeng Y, Xuedan Y, Biao G, Yibing T, et al. Measles mass campaign experience and effect evaluation of Guizhou province, 2003-2004. Chinese Journal of Vaccines and Immunization. 2006;12(1):7-12.

11. Uzicanin A, Eggers R, Webb E, Harris B, Durrheim D, Ogunbanjo G, et al. Impact of the 1996-1997 supplementary measles vaccination campaigns in South Africa. Int J Epidemiol. 2002;31(5):968-76.

12. Khetsuriani N, Deshevoi S, Goel A, Spika J, Martin R, Emiroglu N. Supplementary immunization activities to achieve measles elimination: experience of the European Region. J Infect Dis. 2011;204 Suppl 1:S343-52.

13. Dabral M. Cost-effectiveness of supplementary immunization for measles in India. Indian Pediatr. 2009;46(11):957-62.

14. Castillo-Solorzano CC, Matus CR, Flannery B, Marsigli C, Tambini G, Andrus JK. The Americas: paving the road toward global measles eradication. J Infect Dis. 2011:204 Suppl 1:S270-8.

15. Parker Fiebelkorn A, Redd SB, Gallagher K, Rota PA, Rota J, Bellini W, et al. Measles in the United States during the postelimination era. J Infect Dis. 2010;202(10):1520-8
16. Strebel PM, Henao-Restrepo AM, Hoekstra E, Olive JM, Papania MJ, Cochi SL. Global measles elimination efforts: the significance of measles elimination in the United States. J Infect Dis. 2004;189 Suppl 1:S251-7.

17. Hinman AR, Orenstein WA, Papania MJ. Evolution of measles elimination strategies in the United States. J Infect Dis. 2004;189 Suppl 1:S17-22.

18. Katz SL, Hinman AR. Summary and conclusions: measles elimination meeting, 16-17 March 2000. J Infect Dis. 2004;189 Suppl 1:S43-7.

19. Conrad JL, Wallace $\mathrm{R}$, Witte JJ. The epidemiologic rationale for the failure to eradicate measles in the United States. Am J Public Health. 1971;61(11):2304-10.

20. Hu Y, Li Q, Luo S, Lou L, Qi X, Xie S. Timeliness vaccination of measles containing vaccine and barriers to vaccination among migrant children in East China. PLoS One. 2013;8(8):e73264.

21. Chao M, Lixin $H$, Jin M, Yan Z, Lei $C$, Xiaofeng $L$, et al. Measles epidemiological characteristics and progress of measles elimination in China, 2010. Chinese Journal of Vaccines and Immunization. 2011;17(3):242-5 (In Chinese).

22. NHFPC C. Report on China's migrant population development. 2013. [http://www nhfpc.gov.cn/ldrks/s7847/201309/12e8cf0459de42c981c59e827b87a27c.shtml] (Accessed on 23 Jan 2015).

23. van den Hof S, Berbers GAM, de Melker HE, Conyn-van Spaendonck MAE. Seroepidemiology of measles antibodies in the Netherlands, a cross-sectional study in a national sample and in communities with low vaccine coverage. Vaccine. 1999;18(9-10):931-40.

24. DeSerres G, Joly JR, Fauvel M, Meyer F, Masse B, Boulianne N. Passive immunity against measles during the first 8 months of life of infants born to vaccinated mothers or to mothers who sustained measles. Vaccine. 1997;15(6-7):620-3.

25. Janaszek W, Slusarczyk J. Immunity against measles in populations of women and infants in Poland. Vaccine. 2003:21(21-22):2948-53.

26. Cutts FT, Izurieta HS, Rhoda DA. Measuring coverage in MNCH: design, implementation, and interpretation challenges associated with tracking vaccination coverage using household surveys. PLoS Med. 2013;10(5):e1001404.

27. Orenstein WA. The role of measles elimination in development of a national immunization program. Pediatr Infect Dis J. 2006:25(12):1093-101.

28. Cohen BJ, Parry RP, Doblas D, Samuel D, Warrener L, Andrews N, et al. Measles immunity testing: comparison of two measles IgG ELISAs with plaque reduction neutralisation assay. J Virol Methods. 2006;131(2):209-12.

\section{Submit your next manuscript to BioMed Central and take full advantage of:}

- Convenient online submission

- Thorough peer review

- No space constraints or color figure charges

- Immediate publication on acceptance

- Inclusion in PubMed, CAS, Scopus and Google Scholar

- Research which is freely available for redistribution 